\title{
Subject Index Vol. 65, 1995
}

Activity pattern 110

Adapids 59

Agonistic interactions 163

Alarm calls 229

Allometry 181

Alouatta caraya 115

Anterior dentition 59

Anthropoid origins 59

Anticipation 34

Ateles 85

Athene cunicularia 229

Baboon 202

Behavioral enrichment 214 Brazil 115,224 Bushbaby 110

Cage litter 38

Calcification 1

Callicebus cupreus 118,210

personatus 224 Calling frequency 110 Callithrix aurita 25

jacchus 229 Callitrichidae 25, 48, 229 Canine honing 59 Captivity 214 Capuchin 234

monkey(s) 38,54 Carrying behaviour 210 Ceboidea 59

Cebus apelía 54, 234

- $\quad$ capucinus 38,159

Cercopithecoidea 59

Cerrado 115

Chimpanzee(s) 1,43,174 Chromosome-banding

patterns 54 Chromosomes 100 Condylar canal 144 Conflict resolution 14 Conservation genetics

154 Constitutive heterochro-

matin 54

Daily path length 224 Dentition 1 Dependency 34 Diaphyseal morphology 181 DNA 154

- $\quad$ fingerprinting 174

Dominance 30

Dry season resource 115

Eirabarbara 229 Emissary veins 144 Environmental enrichment

38 Evolution 121

Facial displays 121 Feeding behavio(u)r 43,85, 115

priority 163

strategies 159 Female dominance 163 Field study 118,169 Foraging 85

Fossil primates 59 Frustration 34

Galago 110 Genetic diversity 154 Gestation period 210 Golden monkeys 138 Gombe National Park 43 Gorilla 144 
gorilla beringei 14

- gorilla 219 Great apes 1 Grooming 30

Haptic perception 202 Hemispheric asymmetry 202 Home range 224 Hominid 234 Hominoid 1

Hominoidea 59 Homohabilis 234 Homology 121 Howler monkeys 115 Human predation 172

Inclusive fitness 174 Infant parking 172

- $\quad$ survival 118

Infanticide 169

Interbirth intervals 210

Inteposition during conflict

14 Interspecific interaction 169

Karyotype 100 Kinship 174

Ladders 219 Leaf monkeys 138 Leaping frequency 181

style 181 Lemurs 163,169 Leontopiíhecus 48

rosalia 229 Lethargy 214 Liontamarins 48 Loris 100 Lowland gorillas 219

Macaca arctoides 34

- fascicularis 30

Macaques 30, 121

Madagascar 169

Male genital pathology 48 Male-male aggression 14

- $\quad$ competition 14

Manual strategies 202

Marmosets 25, 229

Marsh habitat 115

Maternal behavio(u)r 169,

172 Mating systems 25 Meat-eating 43 Mentawai Islands 172 Mustelidae 229

Notharctus 59

Orangutan(s) 1,214

Pan 144

- $\quad$ troglodytes 43,174

Papionini 121

Paternity 174

Peat-bathing 38

Pelage maintenance 38

Peru 118

Phylogenetic reconstruction

121 Phytogeny 138 Pig-tailed langur 172 Polygyny 25 Polymoøhism 100 Pongo 144

- $\quad$ pygmaeus 214

Predation 169,229

Premolar mophology 59

Prostate gland 48

Prostatic hype $\varphi$ lasia 48

Psychological well-being 214

Radiography 1

Random amplified polymorphic DNA 154

Range use 85

Ranging behaviour 224 
rDNA 138

Reproduction 118

Reproductive parameters 210

Resource competition 159

Restriction maps 138

Retroarticular foramen 144

Rhinopithecus 154

Ruffed lemurs 163

Scavenging 43 Scramble competition 159 Separation anxiety 34 Sexual dimophism 59 Sigmoid sinus 144 Silent bared-teeth display

121 Simias concolor 172 Slender loris 100 Snub-nosed monkey 154 Social behavior 163,174 organisation 25

rank 30 Socioecology 159 Sponges 219 Sticks 219 Stone tools 234 Strepsirhines 181 Strigidae 229 Stumptailed macaque 34 Subspecies 100

Tamarins 229

Tanzania 43

Task performance 34

Taxonomy 100

Teeth 1

Temporal sinus 144

Tension reduction 14

Thermoregulatíon 38

Throwing 234

Titi monkey(s) 118, 210, 224

Tool making 219

- $\quad$ use 219

Tooth development 1 Twinning 118

Varecia variegata 163 Vocalization 110

White-faced capuchins 159 Within-group competition 159

Yellow fever 110 